\title{
IMPLICATION OF AFLATOXINS AS POTENT CARCINOGENS
}

\author{
Fatima Mukhtar \\ Department of Microbiology, Umaru Musa Yar'adua University Katsina State, Nigeria.
}

\begin{abstract}
Aspergillus species influence human and animal health directly and indirectly with a significant economic impact on the society. A. flavus and A. parasiticus are the two major species that produce aflatoxins. Several mycotoxins are reported from several other mycotoxigenic fungi of which the aflatoxins are the most toxic and damaging polyketides. Economically important crops such as maize, rice, cotton seed, peanuts, and spices are all susceptible to contamination of aflatoxin. The aim of this review is to make an extensive review and come up with ways to curtail this global challenge on how to manage aflatoxin contamination in crops and other food products since the toxins have been classified as potent carcinogens and about $25 \%$ of food is being lost due to aflatoxin contamination annually. The International Agency for Research on Cancer (IARC) conducted evaluation of several chemicals of their carcinogenic potential and classified aflatoxins as most potent natural, known human carcinogens. The methodology was by selecting the most recent researches conducted on new techniques to be employed in overcoming the issue of aflatoxin contamination both during the pre and post-harvest strategies. The Data gathered was subjected to appropriate statistical tool so as to come up with more improved techniques. It was found that both chemical and physical methods have certain drawbacks which may lead to drop in human and animal health, cause a significant decline in the quality of food products, losses of nutritional value, high cost and cause undesirable health effects, but biological methods using microorganisms seems more promising, they provide an attractive alternative tool for removing toxins and safeguarding the value of food and feed in an eco-friendly way. Key words: Aflatoxin, Contamination, Crops, Pre and Post-harvest.
\end{abstract}

\section{INTRODUCTION}

Mycotoxins are a much diversified group of toxic compounds produced by spore-forming fungi, known to cause noxious effects to the health of human and animals. Food security is regularly risked by mycotoxins appearing in food (Ilesanmi and Ilesanmi, 2011).

Amongst the mycotoxins, aflatoxins are most intensively sought because of their immunotoxicity acting on phagocytes and cellmediated immunity. They are considered as natural contaminants of many food products and feeds (Ilesanmi and Ilesanmi, 2011).

Identification of aflatoxin was linked to a groundnut meal contaminated with $A$. flavus leading to mysterious disease "Turkey $\mathrm{X}$ disease" that killed thousands Turkey poultry birds in England in 1960's. The toxic principles were named as aflatoxins (A.flavus toxins) (PACA, 2015). Aflatoxin $B 1$ was identified as the most powerful and lethal naturally occurring liver carcinogen. The atmospheric conditions conducive for aflatoxin production are high moisture during harvest, dry weather near crop maturity and inadequate drying and storage of crops (Wacoo et al., 2014). Post harvesting conditions such as transport, storage (excess heat and moisture, pest related damage, long periods of storage) and food processing influence the production of aflatoxins. Aflatoxin contamination is also influenced by environmental factors such as geography, and agriculture/ agronomic practices (PACA, 2015).

Aspergillus species influence human and animal health directly and indirectly with a significant economic impact on the society. $A$. flavus and $A$. parasiticus are the two major species that produce aflatoxins. Several mycotoxins are reported from several other mycotoxigenic fungi of which the aflatoxins are the most toxic and damaging polyketides (Usha, 2010). Economically important crops such as maize, rice, cottonseed, peanuts, and spices are all susceptible for contamination of aflatoxin. $A$. flavus is the major contributor of aflatoxin in pre and post-harvest agricultural food and feed. 
Special Conference Edition, November, 2019

It is a major global challenge to manage Aspergillus infections in humans and aflatoxin contamination in crops and other food products (Bhetariya et al., 2011). They are toxic to human and animal health. They cause liver and kidney damage, cause immunosuppressive, highly carcinogenic and mutagenic effects, they can accumulate through the food chain posing a serious health concern to both humans and animals and have been associated with various diseases, such as aflatoxicosis (Usha, 2010). The presence of aflatoxins especially aflatoxin B1 in food such as peanuts, milk, and corn can increase a person's risk of liver cancer. Production of aflatoxin is optimal at relatively high temperatures, so contamination is most acute and widespread in warm, humid climates of the tropical and subtropical regions of the world aflatoxins have also been found in temperate countries of Europe and North America (PACA, 2015).

There have been identified 18 types of aflatoxins, nevertheless, the naturally occurring and well-known ones are aflatoxin B1 (AFB1), aflatoxin B2 (AFB2), aflatoxin G1 (AFG1) and aflatoxin G2 (AFG2). These names were given due to their blue (B) or green (G) fluorescence properties under ultraviolet light and their migration patterns during chromatography (Wacoo et al., 2014).

The International Agency for Research on Cancer (IARC) conducted evaluation of several chemicals of their carcinogenic potential and classified aflatoxins as most potent natural, known human carcinogens. In view of toxic and carcinogenic effects of aflatoxin contaminated foods, US department of Agriculture (USDA) and Food and Drug Administration (FDA) set the tolerance limit of $20 \mathrm{ppb}$ on foods. EU countries allow much lower ppb concentration of Aflatoxins. Accepted levels for toxins are variable for various foods in different countries (Bhetariya et al., 2011).

\section{Toxicity and Mechanism of Action of} Aflatoxins

The aflatoxin B1 is considered to be responsible for both toxicity as well as carcinogenicity. It was classified as group I carcinogen by the International Agency for Research on cancer (Peromingo et al., 2016). Epidemiological data from studies in African countries, particularly in South Africa, South East Asia and India implicate aflatoxins in the hepatobiliary carcinoma, malnutrition, kwashiorkar and marasmus (Malik et al., 2014). Aflatoxins are clearly associated with aflatoxicosis, chronic aflatoxicosis leads to cancer, immune suppression and other, slow pathological conditions, whereas acute aflatoxicosis leads to death in the humans, livestock and domestic animals. All the types of aflatoxins are lipolytic in nature and are easily absorbed across cell membranes from the site of exposure such as gastrointestinal, respiratory tracts and enter into the blood stream, then spread to various tissues and to the liver. Liver is the main target organ and liver damage occurred as a result of consumption of food which is contaminated with aflatoxin B1. As the liver is a lipophilic organ, it stores and concentrates all compounds carried by blood stream, i.e. drugs, contaminants, mycotoxins etc., in the hepatocytes and with a long exposure time, may transform themselves into a cancer cell line (Santini and Retieni, 2013).

Aflatoxins are converted to the reactive 8,9epoxide form (also known as aflatoxin-2,3 epoxide) which is capable of binding to both DNA and proteins by cytochrome P450 enzymes. The reactive aflatoxin epoxide binds to the N7 position of guanines. A reactive glutathione Stransferase system found in the cytosol and microsomes catalyzes the conjugation of activated aflatoxins with reduced glutathione, leading to the excretion of aflatoxins (Santini and Retieni, 2013).

Cytochrome P450 microsomal enzyme converts AFB1 to an epoxide which binds to DNA and albumin in the blood, forms an adduct leading to DNA damage (Qureshi et al., 2015). Moreover, aflatoxin B1-DNA adducts can result in GC to TA transversions. The epoxide preferentially binds to mitochondrial DNA resulting in hepatocarcinogenesis. The binding of AFB1 to DNA at guanine site in liver cells affect the genetic code of enzymes which regulate cell growth (Dohnal et al., 2014). This results in formation of tumors. Aflatoxins are known to bind and interfere with enzymes and substrates that are needed in the initiation, transcription and translation processes involved in protein synthesis by forming adducts with DNA, RNA and proteins. The LD50 values for aflatoxin B1 and $\mathrm{M} 1$ are $\leq 18$ and $12-16 \mathrm{mg} / \mathrm{kg}$ bodyweight respectively (Sharma and Parisi, 2017).

\section{Potency of Aflatoxins as potent carcinogens Carcinogenicity}

Aflatoxins are known to be human carcinogens based on sufficient evidence of carcinogenicity from studies in humans. Aflatoxins were listed in the First Annual Report on Carcinogens as reasonably anticipated to be human carcinogens based on sufficient evidence of carcinogenicity from studies in experimental animals and limited evidence of carcinogenicity from studies in humans; 
Special Conference Edition, November, 2019

however, the listing was revised to known to be human carcinogens in the Sixth Annual Report on Carcinogens in 1991(IARC, 2002).

\section{Studies on mechanisms of carcinogenesis}

Aflatoxin causes genetic damage in bacteria, in cultured cells from humans and experimental animals exposed to aflatoxin invivo. Types of genetic damage observed include formation of DNA and albumin adducts, gene mutations, micronucleus formation, sister chromatid exchange, and mitotic recombination. Metabolically activated aflatoxin B1 specifically induced $G$ to $T$ transversion mutations in bacteria. $G$ to $T$ transversions in codon 249 of the p53 tumor-suppressor gene have been found in human liver tumors from geographic areas with high risk of aflatoxin exposure and in experimental animals (Ostry et al., 2017). In humans and susceptible animal species, aflatoxin B1 is metabolized by cytochrome P450 enzymes to aflatoxin-8,9-epoxide, a reactive form that binds to DNA and to albumin in the blood serum, forming adducts. Comparable levels of the major aflatoxin B1 adducts (the N7guanine and serum albumin adducts) have been detected in humans and susceptible animal species. The 8,9-epoxide metabolite can be detoxified through conjugation with glutathione, mediated by the enzyme glutathione Stransferase (GST). The activity of GST is much higher (by a factor of 3 to 5) in animal species that are resistant to aflatoxin carcinogenicity, such as mice, than in susceptible animal species, such as rats. Humans have lower GST activity than either mice or rats, suggesting that humans are less capable of detoxifying aflatoxin-8,9epoxide. In studies of rats and trout, treatment with chemo preventive agents reduced the formation of aflatoxin B1- guanine adducts and the incidence of liver tumors (IARC, 2002).

\section{Cancer Studies in Humans}

Early evidence for the carcinogenicity of aflatoxins in humans came from epidemiological studies (a case-control study and descriptive studies) that correlated geographic variation in aflatoxin content of foods with geographic variation in the incidence of liver cancer (hepatocellular carcinoma, or primary liver-cell cancer). Studies in Uganda, Swaziland, Thailand, Kenya, Mozambique, and China demonstrated strong significant positive correlations between estimated aflatoxin intake or aflatoxin levels in food samples and the incidence of liver cancer. In the United States, a $10 \%$ excess of primary liver-cell cancer was observed in the Southeast, where the estimated average daily intake of aflatoxin was high, compared with the North and West areas with low aflatoxin intake. In a casecontrol study in the Philippines, levels of aflatoxin in the diets of individuals were estimated retrospectively and the risk of liver cancer increased significantly with increasing estimated aflatoxin consumption. Interpretation of these studies is complicated by potential confounding due to hepatitis B virus infection which is endemic in many of the study areas and is known to cause primary liver-cell cancer (IARC, 2002). In studies that took into account the prevalence of chronic hepatitis $B$ infection, aflatoxin exposure remained strongly associated with liver cancer. Chinese studies in which the prevalence of chronic hepatitis B did not appear to fully explain differences in rates of primary liver-cell cancer were reviewed, and it was concluded that the remaining variance in livercancer incidence was related both to estimated dietary levels of aflatoxins and to measured levels of aflatoxins and their metabolites in the urine. In a study in Swaziland, estimated aflatoxin intake based on levels in food samples was strongly correlated with liver-cancer incidence; in this study, geographic variation in aflatoxin exposure better explained the variation in liver-cancer incidence than did variation in the prevalence of hepatitis B infection (IARC 2002).

The International Agency for Research on Cancer concluded in 1987 that, there was sufficient evidence in humans for the carcinogenicity of naturally occurring aflatoxins (IARC, 2002). This conclusion was reaffirmed in two subsequent reevaluations (IARC, 2002). These reevaluations considered the results of several cohort studies in China and Taiwan, which reported associations between biomarkers for aflatoxin exposure (aflatoxin metabolites in the urine and aflatoxin-albumin adducts in the blood) and primary liver-cell cancer.

\section{Cancer studies on experimental animals}

Aflatoxins caused tumors in several species of experimental animals, at several different tissue sites and by several different routes of exposure. Oral administration of aflatoxin mixtures or aflatoxin B1 alone (in the diet by stomach tube or in the drinking water) caused liver tumors (hepatocellular or cholangiocellular tumors) in all species tested except mice; these included rats, hamsters, marmosets, tree shrews, and monkeys. In addition, kidney (renal-cell) and colon tumors occurred in rats, benign lung tumors (adenoma) in mice, and tumors of the liver, bone (osteogenic sarcoma), gallbladder, and pancreas (adeno carcinoma) in monkeys. 
Special Conference Edition, November, 2019

When administered by intraperitoneal injection, aflatoxin B1 caused liver tumors in infant mice, adult rats, and toads. Aflatoxin B1 administered by intraperitoneal injection to pregnant and lactating rats caused tumors of the liver, digestive tract, urogenital system, and nervous system in the mothers and offspring. Aflatoxin mixtures administered by subcutaneous injection caused tumors at the injection site (sarcoma) in rats and mice. Aflatoxins B2, G1, and M1 also caused liver tumors in experimental animals, but generally at lower incidences than did aflatoxin mixtures or aflatoxin B1 alone. In rats, aflatoxin G1 also caused kidney tumors when administered orally and a low incidence of injection site tumors (sarcoma) when administered by intraperitoneal injection. Both enhancement and inhibition of aflatoxin's carcinogenicity were observed following coadministration of aflatoxins with various diets, viruses, parasites, known carcinogens, and other chemicals (Ostry et al., 2017). IARC (2002) concluded that there was sufficient evidence in experimental animals for the carcinogenicity of naturally occurring mixtures of aflatoxins and aflatoxins $\mathrm{B} 1, \mathrm{G} 1$, and $\mathrm{M} 1$; limited evidence for the carcinogenicity of aflatoxin B2; and inadequate evidence for the carcinogenicity of aflatoxin G2. In its 2002 evaluation, IARC reported on several more recent studies suggesting that experimental animals infected with hepatitis B virus (woodchucks, tree shrews, and transgenic mice heterozygous for the p53 tumor-suppressor gene) were more sensitive to the carcinogenic effects of aflatoxin than uninfected animals. IARC (2002) concluded that these studies confirmed the carcinogenicity of aflatoxins in experimental animals.

\section{Methods of detecting Aflatoxin}

Detection methods of aflatoxin has underwent remarkable development since its discovery. Thin layer chromatography is one of the oldest techniques used to analyze contaminated samples (Naaz et al., 2014). Other methods are also used such as high performance liquid chromatography with fluorescent detector or with fluorimetric detector. Aflatoxins are also detected by liquid chromatography coupled to a mass spectrometer (Andrade et al., 2013). Other methods of detection were elaborated such as immune-affinity column immune-enzymatic and immunochemical methods ((Naaz et al., 2014). Detection of ultra-traces of Aflatoxin is extremely important for food safety, this detection requires very powerful techniques. Among the preview of these techniques during this bibliographic research, an aptasensor using unmodified gold nanoparticles indicator based on the aggregation phenomenon of gold nanoparticles induced by salt was developed (Luan et al., 2015). The detection limit is $3.5 \mathrm{pg} / \mathrm{mL}$. In addition, the immunosensor was successfully applied for determination of AFB1 in corn powder, which showed a good correlation with the results obtained by high performance liquid chromatography (Zang et al., 2016).

Technique ultra-high pressure liquid chromatography tandem mass spectrometry was developed to identify and quantify simultaneously mycotoxins in ensiling grasses. It was performed using a modified QuEChERS extraction by employing an acidified aqueous extraction (McElhinney et al., 2015). Another uncomplicated technique with a detection limit of $0.03 \mathrm{ng} / \mathrm{mL}$ for AFB1 based on sensitive surfaceenhanced Raman scattering beacons has been developed without nucleic acid amplification (Zhao et al., 2015).

Other ultra-sensitive strategies; colorimetric and homogeneous for AFB1 detection were set up using a DNA aptamer, and two halves of split DNAzyme has been developed (Seok et al., 2015).

\section{Detoxification of Aflatoxins in Foods}

Different methods employed for decontamination/detoxification of aflatoxins include physical, chemical or biological methods. An ideal detoxification method must ensure that the degradation process maintains the nutritive value of food and feed, will not result in the introduction of new toxic or carcinogenicmutagenic substances and the process must also destroy Aspergillus spores and mycelia, preventing the proliferation and production of new toxins under favorable conditions (Filbert and Brown, 2012).

\section{Physical Methods}

Physical processes involve the separation of the contaminated fractions, removal or inactivation of aflatoxins by physical means, such as heat, cooking, roasting, and radiation (Hwang and Lee, 2006). Aflatoxins can be separated from the feed by cleaning. Cleaning is a multistep process which removes the dust, husks and products colonized by molds by mechanical sorting and washing. Seperation of discolored seeds/kernels also minimizes the aflatoxin contamination. Aflatoxins have low solubility in water. Hence washing may not remove the aflatoxins from the feeds. However, Hwang \& Lee (2006) reported $40 \%$ aflatoxin reduction in contaminated wheat by washing. 
Special Conference Edition, November, 2019

Heating is another method of destroying aflatoxins. AFs have high decomposition temperatures ranging from $237^{\circ} \mathrm{C}-306^{\circ} \mathrm{C}$. Various heat treatments such as boiling, roasting, baking and steaming provide a viable mechanism for reducing the AFs concentration in foodstuffs (Jalili, 2015). Ionizing radiation such a gamma radiation had little effect when used directly in detoxifying the aflatoxins. It indirectly decontaminates the aflatoxins by radiolysis of water, which generates free radicals. (Ozkan et al., 2015).

Adsorption is another method of aflatoxin reduction. It involves the binding of toxin compound to the adsorbent compound during digestion in gastro intestinal tract (Bennett and Kale, 2007).

\section{Chemical Methods}

The effective chemical agents that degrade aflatoxins include:

a) Chlorinating agents such as sodium hypochlorite, chlorine dioxide, and gaseous chlorine

b) Oxidizing agents such as hydrogen peroxide, ozone, and sodium bisulphate and

c) The hydrolytic agents' acids and alkalis. These chemicals either oxidize the double bond of the terminal furan ring or hydrolyze and oxidize the lactone ring of AFB1.

Other chemicals that are tested for aflatoxin degradation are solutions with $75 \%$ methanol, $5 \%$ dimethylamine hydrochloride, aldehydes, benzoyl peroxide, osmium tetroxide, iodine, ferrous ammonium sulphate, potassium permanganate, quinones, sodium borate or formaldehyde. But their use in aflatoxin degradation in foods is limited due to the problems associated with their residues (Wacoo et al., 2014).

\section{Biological Methods}

Many bacteria, yeast and fungi are able to degrade aflatoxins in solutions. Flavobacterium aurantiacum NRRL B-184, that could irreversibly remove aflatoxin $B 1$ from aqueous solution has been reported by Sowley (2016). The bacterium has completely detoxified the toxin contaminated milk, oil, peanut butter, peanuts and corn, whereas it partially detoxified the contaminated soya bean, also the bacterium $F$. aurantiacum successfully removed the aflatoxin M1 from naturally contaminated milk.A quantity $9.9 \mu \mathrm{g} / \mathrm{ml}$ of aflatoxin was completely removed at a cell concentration of $7.0 \times 10^{3}$ cells $/ \mathrm{ml}$ at $30^{\circ} \mathrm{C}$ after 4 hours of incubation (Sowley, 2016). Other microorganisms capable of converting aflatoxin into aflatoxicol and other compounds include Corynebacterium rubrum, Aspergillus Both viable and non-viable cells of lactic acid bacteria have the same adsorbent ability to bind niger, Trichoderma viride, Mucor ambiguus, Dactylium denroides, Mucor griseocyanus, Absidia repens, Helminthosporium sativum, Mucor alternans, Rhizopus arrhizus, Rhizopus oryzae, Rhizopus stolonifer, and the protozoan Tetrahymena pyriformis (Sharma and Parisi, 2017).

\section{DISCUSSION}

Harmful effects caused by this dangerous toxin have directed researchers towards finding new strategies for prevention and detoxification in order to preserve the safety of products intended for human consumption (Ben SalahAbbes et al., 2015). The efficiency of aflatoxin detoxification relies on different factors, including food conditions (food constituents, moisture content and $\mathrm{pH}$ conditions), detoxification technologies and conditions (Pankaja et al., 2018). Therefore, several approaches have been applied to detoxify AFs in crops and during postharvest (Spadaro \& Garibaldi, 2017) as physical, chemical and biological methods (Siciliano et al., 2016). The chief problems with chemical and physical methods are their restricted range of effect on different mycotoxins and some chemicals may lead to drop in animal health. Moreover, there is a common drawback associated with physical and chemical treatments, since they may also cause a significant decline in the quality of food products, losses of nutritional value, high cost and cause undesirable health effects (Prettl et al., 2017).

Over the past decades. The use of selected microorganism to control mycotoxification and postharvest disease has greatly increased, providing an attractive alternative tool for removing toxins and safeguarding the value of food and feed (Wambacq et al., 2016). Biological control provides safe methods for removing aflatoxins from food (Fan et al., 2013). Several studies have reported the capability of many microorganisms including bacteria, yeast, fungi, actinomycetes and algae in removing or degradation of aflatoxins from both food and feed (Hathout et al., 2014). Among all types of available microorganisms that may be utilized to remove aflatoxin from contaminated medium, lactic acid bacteria (LAB) would be a suitable choice for reducing the bioavailability of aflatoxins because of their unique characteristics, they have large plasmid and mega genome, they are Generally Recognized As Safe (GRAS) by USFDA, also some of them have a beneficial effects on health which are termed probiotics (Fan et al., 2013). AFB1 because adsorption occurs due to the interaction between the toxins and the 
Special Conference Edition, November, 2019 functional groups of the cell surface (Hathout et al., 2014).

\section{CONCLUSION}

Aspergillus species influence human and animal health directly and indirectly with a significant economic impact on the society. The International Agency for Research on Cancer (IARC) has classified aflatoxins as most potent natural known human carcinogens. Exposure to aflatoxins needs to be kept as low as possible to protect the consumer. Many countries have regulations governing aflatoxins in food with prescribed acceptable limits, and most have maximum permitted or acceptable levels for different foodstuffs. Aflatoxins damage health and business opportunities, and importing countries are imposing increasingly more stringent regulations.

Control of aflatoxins requires an integrated approach, whereby aflatoxins are controlled at all stages from the field to the table so as to reduce risk. Such an approach includes targeted

\section{REFERENCES}

Andrade, P.D., da Silva, J.L.G. and Caldas, E.D. (2013). Simultaneous analysis of aflatoxins $B 1, B 2, G 1, G 2, M 1$ and ochratoxin A in breast milk by high-performance liquid chromatography/fluorescence after liquid extraction with low temperature purification (LLE-LTP). J. Chromatogr. 1304: $61-8$.

Bennett, J.W., Kale, S. and Yu, J. (2007). Aflatoxins: Background, Toxicology and Molecular Biology. In: Simjee S (ed.), In: Infectious Disease: Foodborne Diseases. Humana Press Inc., Totowa, NJ, USA, Pp. 355-373.

Bhetariya, P.J, Madan, T., Basir, S.F., Varma, A. and Usha, S.P. (2011). Allergens/Antigens, toxins and polyketides of important Aspergillus species. Indian J. Clin. Biochem. IJCB. 26(2):104-119. doi: 10.1007/s12291-011-0131-5

Dohnal, V., Wu, Q. and Kuca, K. (2014). Metabolism of aflatoxins: key enzymes and interindividual as well as interspecies differences. Arch. Toxicol. 88(9):16351644. doi: $10.1007 /$ s00204-014-1312-9

Fan, Y., L. Zhao, Q. Ma, X. Li and H. Shi (2013). Effects of Bacillus subtilis ANSB060 on growth performance, meat quality and aflatoxin residues in broilers fed moldy peanut meal naturally contaminated with aflatoxins. Food Chem. Toxicol., 59: 748753.

Filbert, M.E. and Brown, D.L. (2012). Aflatoxin Contamination in Haitian and Kenyan Peanut Butter and Two Solutions for Reducing Such Contamination. Journal of plant breeding practices, enhancement of host plant resistance, and biological control methods, coupled with post-harvest technologies such as proper drying and storage of potentially affected crop products, as well as development of appropriate alternative uses to retain at least some economic return on value of damaged crop. Therefore by removing the sources of contamination, promoting better agricultural and storage techniques, ensuring adequate resources are available for testing and early diagnosis, enforcing strict food safety standards, informing and educating consumers and (small/subsistence) farmers, promoting better livestock feeding and management, and creating general awareness about personal protection, are some of the ways in which national authorities can help to control aflatoxins.

The use of biological methods (microorganisms) should be fully employed as they seems more promising, provide an attractive alternative tool for removing aflatoxins and safeguarding the value of food and feed in an eco-friendly way.

Hunger \& Environmental Nutrition, 7 (7): 2-3. https://doi.org/10.1080/19320248.2012.70 $\underline{7109}$

Hathout, A.S. and S.E. Aly, (2014). Biological detoxification of mycotoxins: $A$ review. Ann. Microbiol., 64: 905-919.

Hwang, J. H. and Lee, K.G. (2006). Reduction of aflatoxin B1 contamination in wheat by various cooking treatments. Food Chem. 98(1): 71-75.

IARC. (2002). Aflatoxins In Traditional Herbal Medicines, Some Mycotoxins, Naphthalene and Styrene. IARC Monographs on the Evaluation of Carcinogenic Risks to Humans, vol. 82. Lyon, France: pp. 171366

Ilesanmi, F.F. and Ilesanmi, O.S. (2011). Knowledge of aflatoxin contamination in groundnut and the risk of its ingestion among health workers in Ibadan, Nigeria. Asian Pac. J. Trop. Biomed; 1(6): 493-5.

Jalili, M. (2015) A review on aflatoxins reduction in food. Iranian Journal of Health, Safety and Environment 3(1): 445-459.

Luan, Y., Chen, Z., Xie, G., Chen, J., Lu, A. and Li, C. (2015). Rapid visual detection of aflatoxin $\mathrm{B} 1$ by label-free aptasensor using unmodified gold nanoparticles. J. Nanosci. Nanotechnol. 15(2): 1357-61

Malik, A., Ali S., Shahid, M. and Bhargava, R. (2014). Occupational exposure to Aspergillus and aflatoxins among foodgrain workers in India. Int. J. Occup. Environ. Health. 20(3):189-193. doi: 10.1179/2049396714Y.0000000055. 
Special Conference Edition, November, 2019

McElhinney, C., O'Kiely, P., Elliott, C. and Danaher, M. (2015). Development and validation of an UHPLC-MS/MS method for the determination of mycotoxins in grass silages. Food Addit. Contam. Part A Chem. Anal Control Expo Risk Assess ; 32(12): 2101-12.

Naaz, F., Abdin, M.Z. and Javed S. (2014). Protective effect of esculin against prooxidant aflatoxin B1-induced nephrotoxicity in mice. Mycotoxin Res. 30(1): 25-32

Ostry, V., Malir, F., Toman, J. and Grosse, Y. (2017). Mycotoxins as human carcinogens the IARC Monographs classification. Mycotoxin research, 33(1), 65-73.

Ozkan, A., Bindak, R. and Erkmen, O. (2015). Aflatoxin $\mathrm{B}(1)$ and aflatoxins in ground red chilli pepper after drying. Food Addit. Contam. Art B Surveill.;8(3):227-233. doi: 10.1080/19393210.2015.1063014.

PACA (2015) Aflatoxin Impacts and Potential Solutions in Agriculture, Trade and Health. An Introduction to Aflatoxin Impacts in Africa IARC Monograph 82. Aflatoxins Pp. 172-300.

Pankaja, S.K., Shib, Hu, and Kevin, M. K. (2018) A review of novel physical and chemical decontamination technologies for aflatoxin in food. Trends Food Sci. Technol. 71, 7383.

Peromingo, B., Rodriguez, A., Bernaldez, V., Delgado, J. and Rodriguez, M. (2016). Effect of temperature and water activity on growth and aflatoxin production by Aspergillus flavus and Aspergillus parasiticus on cured meat model systems. Meat Sci.;76-83. doi: 10.1016/j.meatsci.2016.07.024.

Prettl, Z., Dési, E., Lepossaa, A., Kriszt, B., Kukolya, J. and Nagya, E. (2017) Biological degradation of aflatoxin $\mathrm{B} 1$ by $\mathrm{a}$ Rhodococcus pyridinivorans strain in byproduct of bioethanol. Anim. Feed Sci. Technol. 224, 104-114.

Qureshi, H., Hamid, S.S., Ali S.S., Anwar, J., Siddiqui, A.A. and Khan, N.A. (2015). Cytotoxic effects of aflatoxin B1 on human brain microvascular endothelial cells of the blood-brain barrier. Med. Mycol. ;53(4):409-416. doi: $10.1093 / \mathrm{mmy} / \mathrm{myv} 010$.

Santini, A. and Ritieni, A. (2013). Aflatoxins: Risk, exposure and remediation. In: Aflatoxins: Recent advances and future prospects.
Razzaghi- Abyaneh M (Ed.) In Tech Open, UK, Pp. 343-376

Seok, Y., Byun, J.Y., Shim, W.B. and Kim, M.G. (2015). A structure-switchable aptasensor for aflatoxin B1 detection based on assembly of an aptamer/split DNAzyme. Anal. Chim. Acta. ; 886: 182-7.

Sharma, R.K., and Parisi, S. (2017). Toxins and contaminants in Indian food products. Springer International Publishing AG, Switzerland, Pp. 13-24.

Siciliano, I., Spadaro, D., Prelle, A., Vallauri, D., Cavallero, M., Garibaldi, A. and Gullino, M. (2016) Use of cold atmospheric plasma to detoxify hazelnuts from aflatoxins. Toxins (Basel), 8, 125-204

Sowley, E.N.K (2016). Aflatoxins: A silent threat in developing countries. Afr. J. Biotechnol. 159(35): 1864-1870.

Spadaro, D. and Garibaldi, A. (2017) Containment of mycotoxins in the food chain by using decontamination and detoxification techniques. In: "Practical Tools for Plant and Food Biosecurity".

Tamiya, H., Ochiai, E., Kikuchi, K., Yahiro, M., T. and Watanabe, A. (2015). Secondary metabolite profiles and antifungal drug susceptibility of Aspergillus fumigatus and closely related species, Aspergillus lentulus, Aspergillus udagawae, and Aspergillus viridinutans. J. Infect. Chemother. Off. J. Jpn. Soc. Chemother. 21(5):385-391.

doi: 10.1016/j.jiac.2015.01.005

Usha, P. (2010). Fascinating potential of Aspergilli. Indian J. Clin. Biochem. IJCB. 25(4):331334. doi: 10.1007/s12291-010-0079-x.

Wacoo, A.P, Wendiro, D., Vuzi, P.C. and Hawumba, J.F. (2014). Methods for Detection of Aflatoxins in Agricultural Food Crops Article ID 706291, Pp. 15.

Zhang, X., Li, C.R., Wang, W.C., Xue, J., Huang, Y.L. and Yang, X.X. (2016). A novel electrochemical immunosensor for highly sensitive detection of aflatoxin B1 in corn using single-walled carbon nanotubes/chitosan. Food Chem.192: 197-202.

Zhao, Y., Yang, Y., Luo, Y., Yang, X., Li, M. and Song, Q. (2015). Double detection of mycotoxins based on SERS labels embedded Ag@Au core-shell nanoparticles. ACS Appl. Mater. Interfaces 7(39): 21780-6. 